\section{Welcome to a new, international open-access research journal with a particular focus on the development of theoretical and experimental techniques and methodology}

\section{Fulvio Melia}

University of Arizona, Tucson, AZ, USA

I have recently had the privilege of being appointed Editor-in-Chief of this very exciting and innovative Open Access Journal, and hereby extend a warm welcome to everyone as we launch Astronomy Studies Development, which will seek to publish high quality, peerreviewed, original manuscripts in all fields of astronomy and astrophysics, though with a particular focus on mathematical techniques and methodology and innovative ideas for instrumental development and modeling in astronomy and astrophysics. The journal will also seek to publish simulations in all areas, including cosmology, particle astrophysics, accretion, and diffuse media. Our journal will include both full length research articles and letter articles, and its coverage extends over solar, stellar, galactic and extragalactic astronomy and astrophysics, and will report original research in all wavelength bands.

Astronomy and Astrophysics are rather mature disciplines, with a history of quality journals over the past century or more. So one may reasonably ask why a new journal such as this is needed. Obviously, I myself have answered this question in the affirmative.
After a long career in research and publishing, I have the perspective to provide several good reasons for helping to promote the evolution of publishing in Astronomy and Astrophysics to a place more in line with present technology, taking advantage of the fast paced environment in which we live.

The Open Access format will allow us to publish manuscripts within one-to-two months of submission in an environmentally responsible manner. This format permits the widest possible distribution of published results with a speed and efficiency almost rivaling those of archival preprint servers, though with the greatly added advantage of a thorough refereeing process. It is never easy starting a new journal, but we strongly believe that these factors are among the many that create a niche for this type of medium, and we are placing ourselves at the forefront of open-access publishing in Astronomy and Astrophysics.

This alone is reason enough to make Astronomy Studies Development a viable medium, but there is more. In addition to providing a fast dissemination of refereed research reporting with open access to the world's growing research community, we will also be striving to achieve a reputation for fostering creativity in our authors, encouraging them to explore not only areas within the traditional purview of our field, but also beyond the rather conservative boundaries often imposed by other existing journals.

I have been an editor with well-established journals for many years and am also currently the editor of the theoretical astrophysics series of books at the University of Chicago Press. I don't mind telling you that one of the frustra-
Correspondence: Fulvio Melia, Professor of Physics, Astronomy and Applied Mathematics, University of Arizona, $1501 \mathrm{~N}$ Campbell Ave, Tucson, AZ 85724, USA.

E-mail: eic-asd@physics.arizona.edu

Received for publication: 6 March 2011. Accepted for publication: 7 March 2011.

This work is licensed under a Creative Commons Attribution 3.0 License (by-nc 3.0).

CC Copyright F. Melia, 2011

Licensee PAGEPress, Italy

Astronomy Studies Development 2011; 1:e1 doi:10.4081/asd.2011.e1

tions we've had on the various editorial boards on which I've sat is that well-established journals tend to be ultra-conservative. One of my principal goals with an Open Access Journal such as this is to promote not only timely and important papers, but also to stress the need for new, creative ideas from its authors. Think outside the box! You will find that Astronomy Studies Development will encourage you along the way, maintaining very high standards with rigorous refereeing, but also quite receptive to any innovations that may move the field beyond the current mainstream.

I am delighted to invite you to collaborate with us in this endeavor as an author, referee, and reviewer in your particular area of expertise. I hope very much that you will accept our invitation and look forward to working with you on the creation of innovative, timely, and groundbreaking knowledge. 